\title{
Dynamical modelling of a reactive extrusion process: Focus on residence time distribution in a fully intermeshing co-rotating twin-screw extruder and application to an alginate extraction process
}

\author{
Régis Baron ${ }^{\mathrm{a},{ }^{*}}$, Peggy Vauchel ${ }^{\mathrm{b}, \mathrm{c},{ }^{*},}$, Raymond Kaas $^{\mathrm{a}}$, Abdellah Arhaliass $^{\mathrm{d}}{ }^{\text {, Jack Legrand }}{ }^{\mathrm{d}}$ \\ 1 IFREMER, Dpt. BRM, rue de l'lle d'Yeu, BP 21105, 44311 Nantes Cedex 03, France \\ 2 Univ Lille Nord de France, F-59000 Lille, France \\ ${ }^{3}$ USTL, Laboratoire ProBioGEM, F-59650 Villeneuve d'Ascq, France \\ ${ }^{4}$ GEPEA, Université de Nantes, CNRS, UMR6144, 37 bd de I'Université, BP 406, 44602 Saint-Nazaire Cedex, \\ France \\ *: Corresponding author : Régis Baron, email address : rbaron@ifremer.fr, Peggy Vauchel, email address : \\ peggy.vauchel@polytech-lille.fr
}

\begin{abstract}
:
The context of this study is the modelling of reactive extrusion process based on an alginate extraction protocol. Residence Time Distribution (RTD) is one important part to predict the kinetics of reactive compounds. A simple model is proposed to predict RTD in fully intermeshing co-rotating twin-screw extruders without reaction. This model, which can be easily extended to reactive case in a future work, is based on the extension of an axial dispersion model, including control parameters (screw speed and flow rate) and geometrical parameters (screw profile and die design). Simulations were performed for various operating and geometrical conditions so as to illustrate possibilities offered by the proposed model. Validation was conducted for two different extrusion applications, seaweed extrusion and polymer extrusion. This highlighted the model ability to predict RID for various kinds of materials after adjusting only one parameter thanks to a unique experimental RID curve.
\end{abstract}

Keywords: Alginate, Extraction, Extrusion, Mathematical modeling, Residence time distribution, Simulation, Laminaria digitata

\section{Notation}

$a, b, c$ and $d$ : piecewise constant functions depending on screw geometry ( $\mathrm{m} 6, \mathrm{~m} 3, \mathrm{~m} 3$ and $\mathrm{m} 6$ respectively) (Equation 10)

$C$ : tracer concentration (mol.m-3 or g.m-3),

Cin : input tracer concentration (mol.m-3 or g.m-3)

$D$ : screw diameter $(\mathrm{m})$

Dax : axial dispersion function $\left(\mathrm{m}^{2} . \mathrm{s}-1\right)$

Fdi and Fpi : parameters depending on the channel narrowness in screw zone $i$ (Equation 8)

$\mathrm{Hi}$ : channel height of screw zone $i(\mathrm{~m})$

$i$ : screw zone index (Figure 2)

$j$ : spatial discretization index associated to the last fully filled zone (Figure 2) 
$51 \quad K$ : parameter depending on the die geometry $\left(\mathrm{m}^{3}\right)$ (Equation 9)

$52 \quad l$ : length of fully filled channel (m) (Equation 12)

$53 \quad l_{i}$ : channel length of screw zone $i(\mathrm{~m})$

$54 l_{\text {tot }}$ : total length of the screw channel (partially + fully filled) (m) (Figure 2)

$55 \quad L D P E$ : low density polyethylene

$56 m$ : number of elements for spatial discretization

$57 n$ : number of fully filled screw zones (Figure 2)

$58 n_{t o t}$ : total number of screw zones (Figure 2)

$59 \quad N$ : screw speed $\left(\operatorname{rad} . \mathrm{s}^{-1}\right)$

$60 \quad P$ : pressure $(\mathrm{Pa})$

$61 P_{d}$ : pressure at the head of the die $(\mathrm{Pa})$

$62 Q:$ input flow rate $\left(\mathrm{m}^{3} \cdot \mathrm{s}^{-1}\right)$

$63 Q_{c i}$ : flow rate in the channel of screw zone $i\left(\mathrm{~m}^{3} \cdot \mathrm{s}^{-1}\right)$

$64 Q_{\text {out }}$ : output flow rate $\left(\mathrm{m}^{3} \cdot \mathrm{s}^{-1}\right)$

$65 \quad r$ : algae feed rate to reactive solution feed rate ratio

$66 \quad R T D$ : residence time distribution

$67 S_{i}$ : section of the channel in screw zone $i\left(\mathrm{~m}^{2}\right)$

$68 t$ : time $(\mathrm{s})$

$69 v$ : fluid speed $\left(\mathrm{m} . \mathrm{s}^{-1}\right)$

$70 v_{b x}, v_{b z}:$ boundary values of fluid speed $\left(\mathrm{m} . \mathrm{s}^{-1}\right)$ (Equations 3 and 5)

$71 V_{b}$ : barrel speed $\left({\mathrm{m} . \mathrm{s}^{-1}}^{-1}\right.$ (Figure 1)

$72 V_{i}$ : volume of the channel in screw zone $i\left(\mathrm{~m}^{3}\right)$

$73 W_{i}$ : width of the channel in screw zone $i(\mathrm{~m})$

$74 \quad z$ : abscissa along the unrolled screw channel 
$\alpha_{i}$ and $\beta_{i}$ : parameters depending on the geometry of screw zone $i\left(\mathrm{~m}^{3}\right.$ and $\mathrm{m}^{6}$ respectively) (Equation 8)

$\lambda_{1}$ and $\lambda_{2}$ : correction parameter relative to axial dispersion function $D_{a x}$ in the fully filled zone and in the partially filled zone respectively (Equation 16)

$\mu$ : fluid viscosity (Pa.s)

$\theta_{i}:$ pitch angle in screw zone $i(\mathrm{rad})$

\section{INTRODUCTION}

Extrusion is a continuous process consisting in shaping or in transforming a material within a screw/barrel system. Mostly, the involved mechanisms are purely thermo-mechanical.

Reactive extrusion process, which consists in using extruders as chemical reactors, has developed since few decades. For some applications, it appears as an interesting alternative to batch process, with several advantages due to the fact that it is a continuous process, its modularity (screw profile can be adapted to each application and several zones can be created along the screw to conduct different steps), its thermal regulation facilitated by a favourable surface/volume ratio and its ability to work with high viscosity products, enabling solvent consumption limitation (gains in waste treatment and process safety) (Berzin and $\mathrm{Hu}, 2004$ ). Most of developed reactive extrusion applications deal with polymer science or food fields. Several applications have also been developed with biological raw materials, mostly for biomolecules extraction or for by-products upgrading (Perrin and De Choudens, 1996; N'Diaye et al., 1996; Dufaure et al., 1999; Rouilly et al., 2006). Hence, a previous work highlighted the interest of reactive extrusion process when extracting alginate from brown algae in terms of extraction yield, time, reactant and water demand and alginate rheological properties (Vauchel et al., 2008a). 
Reactive extrusion modelling is essential to help understanding phenomena taking place in the

100 extruder, optimization and scale up. The model structure adopted for alginate extraction 101 application is based on the combination of an extraction kinetics model and one describing material flow inside the extruder. The extraction kinetics model was presented in a previous paper (Vauchel et al., 2008b). The current paper aims at modelling the material flow inside

104 the extruder based on residence time distribution. The coupling between both models will be 105 discussed in a forthcoming paper. Residence time distribution (RTD) is a particularly important parameter in reactive extrusion process as it is directly linked to contact time of reactants. Few authors have proposed models for reactive extrusion processes based on the

108 coupling of a RTD model and kinetics model for chemical reaction and/or for viscosity

109 (Ganzeveld and Janssen, 1993; Prat et al., 1999, 2002; Puaux et al., 2006). RTD models are 110 generally built by fitting experimental RTD curves with different flow models (Ainser, 1996; 111 Puaux et al., 2000). Good correlations have been obtained, especially with the backflow cell 112 model and the axial dispersion model. Nevertheless these models don't directly take into 113 account the geometrical parameters (screws profile and die design) and the control parameters 114 (screw speed and flow rate). These points limit the prediction value of this modelling 115 approach especially when scaling up.

116 The present paper aims at presenting a model enabling to predict the residence time 117 distribution in fully intermeshing twin-screw extrusion process and including all geometrical 118 and control parameters. At first, the physical considerations and hypotheses taken into 119 account are described. Simulations of residence time distribution, in various conditions, are 120 then presented and discussed. The model is then validated in the case of seaweeds and 121 polymer extrusion. 


\section{RESIDENCE TIME DISTRIBUTION MODEL}

\subsection{Seaweeds extrusion process considerations}

127 In seaweeds extrusion application, material flowing in the extruder evolves along the screw

128 channels. In the feeding section, two different phases are injected, a solid one composed of

129 seaweeds cut in pieces, and a liquid one composed of a sodium carbonate solution. However,

130 reaction between seaweeds and reactive solution takes place very rapidly in the extruder

131 (under the combined effects of a sodium carbonate chemical action and the shearing provided

132 by the screws) resulting shortly in a pseudo-homogenous phase. Experiments of instantaneous

133 stop and opening of the extruder showed that it appears in the first third of the screw channels

134 in all cases. This pseudo-homogenous phase is composed of a viscous sodium alginate

135 solution with very small (less than $1 \mathrm{~mm}$ diameter) seaweed particles in suspension. The goal

136 of this work is to build a simple model for RTD prediction. To assure the assumption of a

137 homogenous fluid flowing all along the screw channels, seaweeds under the form of a pseudo

138 homogeneous phase were run twice through the extruder. Tracer experiments for validation,

139 which is presented in a further section, were carried out during the second extrusion run.

140

$141 \quad$ 2.2. RTD model description

142 The spatiotemporal tracer concentration evolution is described by means of an axial

143 dispersion model with two parameters depending on the length of the fully filled channel, the

144 axial dispersion coefficient and fluid speed. The final proposed RTD model is based on the

145 combination of a tracer concentration evolution model and a model for the calculation of the

146 length of a fully filled channel, which are both described below. 


\subsubsection{Length of fully filled channel model}

149 The adopted approach to determine the length(s) of fully filled channel is based on elements

150 described by Baron (1995). A simplified solution of the Navier-Stokes equations is used to

151 describe fluid flow at steady state in a fully intermeshing co-rotating twin-screw extruder. To

152 solve the Navier-Stokes equations, several simplifying assumptions have to be considered

153 concerning extruder geometry, fluid properties and flow type.

154 It is assumed that the screw channel is unrolled and fixed, and that the barrel is plane and

155 slides on the screw channel at $V_{b}$ velocity (Figure 1 ). The totally unrolled channel length $l_{\text {tot }}$ is

156 divided into $n_{t o t}$ zones, corresponding to different screw element geometries composing the

157 screw profile. The correspondence between the abscissa $z$ along the unrolled screw channel

158 and the iteration $i$ for screw zones of different geometries is described in Figure 2. Filling of

159 the screw channel occurs in the opposite direction to the flow (behind the die or reverse pitch

160 screw elements). Hence, it was simpler to perform calculation iteration from the die to the

161 feeding section (see axes directions for $i, j$ and $z$ on Figure 2). The fluid is assumed to be

162 incompressible, Newtonian, and viscosity is assumed to be constant along the screws. The

163 flow is assumed to be established, laminar, isothermal and uniform along the screw channel,

164 which length is considered as infinite (width to length of the channel ratio as well as height to

165 length of the channel ratio are assumed to be close to zero). If the interpenetration zone of the

166 screws is neglected it can be assumed that the channel passes from one screw to the other

167 without leakage or flow restriction. Gravity forces can be neglected compared to others forces

168 as well as inertial forces compared to viscous forces.

169

$$
\left\{\begin{array}{l}
\frac{\partial P}{\partial x}=\mu \cdot \frac{\partial^{2} v_{x}(y)}{\partial y^{2}} \\
\frac{\partial P}{\partial y}=0 \\
\frac{\partial P}{\partial z}=\mu \cdot\left(\frac{\partial^{2} v_{z}(x, y)}{\partial x^{2}}+\frac{\partial^{2} v_{z}(x, y)}{\partial y^{2}}\right)
\end{array}\right.
$$


(2) $\quad v_{x}(y=0)=0$

171

(3) $\quad v_{x}(y=H)=-v_{b x}=-\pi \cdot D \cdot N \cdot \sin \theta$

172

(4) $\quad v_{z}(x, y=0)=0$

173

(5) $\quad v_{z}(x=0, y=H)=v_{b z}=\pi \cdot D \cdot N \cdot \cos \theta$

174

(6) $\quad v_{z}(x=0, y)=0$

175 By doing so, a linear form of the Navier-Stokes equations can be obtained, which can be

176 simplified according to Equation system 1. If the boundary conditions described by Equations

1772 to 6 are taken into account, Equation system 1 can be solved to get fluid speed components

178 expressions according to the solution described by Tadmor and Klein (1970).

(7) $\quad Q_{c}=\int_{0}^{H} \int_{0}^{W} v_{z} \cdot d y \cdot d x$

180

(8) $\quad Q_{c i}=\alpha_{i} \cdot N-\frac{\beta_{i}}{\mu \cdot S_{i}} \cdot \frac{\partial P}{\partial z}$ with $\alpha_{i}=\frac{\pi \cdot D \cdot \cos \theta_{i} \cdot W_{i} \cdot H_{i} \cdot F_{d i}}{2}$ and

$181 \quad \beta_{i}=\frac{\left(W_{i} \cdot H_{i}^{2}\right)^{2} \cdot F_{p i}}{12}$

182 The flow rate in the screw channel at abscissa $z\left(Q_{c}\right)$ is then obtained by solving Equation 7.

183 Its expression (Equation 8) is assumed to be the difference between a pumping flow rate

184 depending on the screw speed $N$ and a drag flow rate depending on the pressure gradient $\frac{\partial P}{\partial z}$

185 (Janssen et al., 1979; Tadmor and Klein, 1970). It holds true for each type of screw element,

186 direct or reverse screw pitch for example. Screw geometry is taken into account via

187 parameters $\alpha$ and $\beta$ (with correction factors $F_{d i}$ and $F_{p i}$ to take into account the impact of a

188 limit layer in the screw channel).

189

(9) $Q_{\text {out }}=\frac{K}{\mu} \cdot P_{d}$ 
(10) $Q_{\text {out }}=\left(\frac{a+b \cdot l \cdot S}{c+\frac{d}{K}+l \cdot S}\right) \cdot N$, with $a=\sum_{i=1}^{n-1}\left(\alpha_{i} \cdot \frac{\beta_{n}}{\beta_{i}}-\alpha_{n}\right) \cdot V_{i}, b=\alpha_{n}$,

$c=\sum_{i=1}^{n-1}\left(\frac{\beta_{n}}{\beta_{i}}-1\right) \cdot V_{i}$ and $d=\beta_{n}$, for $\sum_{i=0}^{n+1} \frac{V_{i}}{S_{i}}<l<\sum_{i=0}^{n} \frac{V_{i}}{S_{i}}$.

192 As the outflow rate $Q_{\text {out }}$ is constrained by the die, it is assumed to follow a Hagen-Poiseuille

193 equation (Equation 9). It depends on the pressure at the end of the die $\left(P_{d}\right)$, which is equal to atmospheric pressure and on the geometrical coefficient $K$, which is inversely proportional to the flow restriction. Moreover, the output pressure of the iteration zone 1 is assumed to be equal to the pressure present at the head of the die, and the output pressure of iteration zone $i$

197 to be equal to the input pressure of the iteration zone $i-1$. Consequently, pressure gradients

198 can be eliminated in the flow rate expression (Equation 8) resulting in the outflow rate expression presented in Equation 10 (Baron, 1995). $a, b, c$ and $d$ are piecewise constant

200 functions, depending on screw geometry.

$$
\frac{d l}{d t}=\frac{Q(t-\tau)-Q_{o u t}}{S_{n}}
$$

202

$$
l=\frac{-a+\left(c+\frac{d}{K}\right) \cdot \frac{Q}{N}}{\left(b-\frac{Q}{N}\right) \cdot S_{n}}
$$

203 Hence, length of the fully filled channel $l$ can be obtained by solving a dynamical equation

204 traducing mass balance (Equation 11). $\tau$ is a pure delay depending on control parameters and 205 length of starved screw $\left(l_{\text {tot }}-l\right)$ and describing the conveying time in the feeding zone. Carrot et 206 al. (1993) proposed a specific flow model for this area. At steady-state, the outflow rate $Q_{\text {out }}$ is 207 equal to the input flow rate $Q$, and the fully filled channel length $l$ can be estimated by 208 Equation 12. 
In the case of a screw profile containing restrictive elements (reverse screw pitch or kneading

210 discs for example), several fully filled channel zones appear along the screws. The proposed

211 model enables to deal with these cases. Two examples are given below, one in the case of a

212 unique fully filled channel zone, the second one in the case of several fully filled channel

213 zones. Screw profiles, corresponding to validation experiments with seaweeds exposed in a

214 further section, are described in Figure 3.

\section{Example 1 (Profile 1)}

216 Screw profile is described in Figure 3a. Each zone corresponds to a different type of screw

217 element and is characterized by four functions depending on its geometry, $l_{i}, S_{i}, \alpha_{i}$ and $\beta_{i}$. In

218 this case, there is only one fully filled zone. Matter fills the screw channel from the die to the

219 feeding zone.

220 If $l<\left(l_{3}+l_{2}+l_{1}\right)$ (Figure 3b), then $a=\left(\alpha_{1} \cdot \frac{\beta_{3}}{\beta_{1}}-\alpha_{3}\right) \cdot S_{1} \cdot l_{1}+\left(\alpha_{2} \cdot \frac{\beta_{3}}{\beta_{2}}-\alpha_{3}\right) \cdot S_{2} \cdot l_{2}$

$221 \quad b=\alpha_{3}$

$222 \quad c=\left(\frac{\beta_{3}}{\beta_{1}}-1\right) \cdot S_{1} \cdot l_{1}+\left(\frac{\beta_{3}}{\beta_{2}}-1\right) \cdot S_{2} \cdot l_{2}$

$223 d=\beta_{3}$.

224 If $\left(l_{3}+l_{2}+l_{1}\right) \leq l<\left(l_{4}+l_{3}+l_{2}+l_{1}\right)($ Figure $3 \mathrm{c})$, then

$a=\left(\alpha_{1} \cdot \frac{\beta_{4}}{\beta_{1}}-\alpha_{4}\right) \cdot S_{1} \cdot l_{1}+\left(\alpha_{2} \cdot \frac{\beta_{4}}{\beta_{2}}-\alpha_{4}\right) \cdot S_{2} \cdot l_{2}+\left(\alpha_{2} \cdot \frac{\beta_{4}}{\beta_{2}}-\alpha_{4}\right) \cdot S_{3} \cdot l_{3}$

$226 \quad b=\alpha_{4}$

$c=\left(\frac{\beta_{4}}{\beta_{1}}-1\right) \cdot S_{1} \cdot l_{1}+\left(\frac{\beta_{4}}{\beta_{2}}-1\right) \cdot S_{2} \cdot l_{2}+\left(\frac{\beta_{4}}{\beta_{2}}-1\right) \cdot S_{3} \cdot l_{3}$

$228 d=\beta_{4}$.

229 And so on ... 
230 For reverse pitch or kneading disc elements, we assumed that they act like a direct pitch

231 element. $\alpha, \beta$ and a mean adjusted section $S$ can be assessed by specific experiments not

232 discussed here.

233 Example 2 (Profile 2)

234 In this case, screw profile contains two restrictive elements zones (Figure 3d), which implies

235 two fully filled zones $\left(l_{2}{ }^{*}, l_{3}{ }^{*}\right)$ on top of the one implied by the die $\left(l_{1}{ }^{*}\right)$. These lengths are

236 characterized by equations similar to equation (13). For $l_{2}{ }^{*}$ and $l_{1}{ }^{*}$, the input flow corresponds

237 to the output flow of the previous fully filled zone but with a specific pure delay. For each

238 fully filled zone $l_{i}^{*}$, functions $a_{i}^{*}, b_{i}^{*}, c_{i}^{*}$ and $d_{i}^{*}$ are defined, just as described above.

239 For example (Figure $3 \mathrm{e}$ ), if $l_{1}^{*}<\left(l_{3}+l_{2}+l_{1}\right)$ then,

240

$a_{1}^{*}=\left(\alpha_{1} \cdot \frac{\beta_{3}}{\beta_{1}}-\alpha_{3}\right) \cdot S_{1} \cdot l_{1}+\left(\alpha_{2} \cdot \frac{\beta_{3}}{\beta_{2}}-\alpha_{3}\right) \cdot S_{2} \cdot l_{2}$

$241 \quad b_{1}^{*}=\alpha_{3}$

$242 \quad c_{1}^{*}=\left(\frac{\beta_{3}}{\beta_{1}}-1\right) \cdot S_{1} \cdot l_{1}+\left(\frac{\beta_{3}}{\beta_{2}}-1\right) \cdot S_{2} \cdot l_{2}$

$243 d_{1}^{*}=\beta_{3}$

244 If $l_{2}^{*}<\left(l_{6}+l_{5}+l_{4}\right)$ then,

$a_{2}^{*}=\left(\alpha_{4} \cdot \frac{\beta_{6}}{\beta_{4}}-\alpha_{6}\right) \cdot S_{4} \cdot l_{4}+\left(\alpha_{5} \cdot \frac{\beta_{6}}{\beta_{5}}-\alpha_{6}\right) \cdot S_{5} \cdot l_{5}$

$246 \quad b_{2}^{*}=\alpha_{6}$

$c_{2}^{*}=\left(\frac{\beta_{6}}{\beta_{4}}-1\right) \cdot S_{4} \cdot l_{4}+\left(\frac{\beta_{6}}{\beta_{5}}-1\right) \cdot S_{5} \cdot l_{5}$

$248 \quad d_{2}^{*}=\beta_{5}$

249 If $l_{3}^{*}<\left(l_{9}+l_{8}+l_{7}\right)$ then, 
$a_{3}^{*}=\left(\alpha_{7} \cdot \frac{\beta_{9}}{\beta_{7}}-\alpha_{9}\right) \cdot S_{7} \cdot l_{7}+\left(\alpha_{8} \cdot \frac{\beta_{9}}{\beta_{8}}-\alpha_{9}\right) \cdot S_{8} \cdot l_{8}$

$b_{3}^{*}=\alpha_{9}$

$c_{3}^{*}=\left(\frac{\beta_{9}}{\beta_{7}}-1\right) \cdot S_{7} \cdot l_{7}+\left(\frac{\beta_{9}}{\beta_{8}}-1\right) \cdot S_{8} \cdot l_{8}$

$d_{3}^{*}=\beta_{9}$

It is easy to generalize Equations 10 and 11 to the case of overlapping fully filled zones.

\subsubsection{Model for tracer concentration}

A largely used approach is based on the description of the flow pattern by conceptual models, combining ideal reactors, which represent the overall features of the physical flow. But intermediate characteristics between those obtained with two ideal limiting cases, the perfect mixer and the plug flow reactor. Therefore, non-ideal models have to be used to describe the material flow. One of the main significant criteria for an extrusion flow model is its ability to backflow cell model (Puaux et al., 2000).

266 In this paper, the axial dispersion model has been chosen. It consists in a combination of the convective transport and an eddy diffusion mechanism in the axial direction.

$$
\text { (13) } \frac{\partial C}{\partial t}=D_{a x} \cdot \frac{\partial^{2} C}{\partial z^{2}}-v \cdot \frac{\partial C}{\partial z}
$$

269 For a constant fluid velocity $v$ and a constant axial dispersion coefficient $D_{a x}$ along the flow axis $z$, the spatio temporal tracer concentration evolution can be described by Equation 13 . 


$$
v(z)=\frac{Q}{S(z)} \text { for } 0<z \leq l
$$

$$
D_{a x}(z)=\lambda(z) \cdot|\alpha(z) \cdot N-Q|
$$

$$
\text { with } \lambda(z)=\lambda_{1} \text { for } 0<z \leq l \text { and } \lambda(z)=\lambda_{2} \text { for } l<z \leq l_{\text {tot }}
$$

275 Equation 13 has been extended to the case where $D_{a x}$ and $v$ functions are piecewise constant.

276 The fluid velocity $v$ depends on the fully filled length $l$ : when the screw channel is partially

277 filled $\left(l<z<l_{t o t}\right)$, it depends on the pumping effect (Equation 14) whilst when the channel is 278 fully filled $(0 \leq z \leq l)$, it depends on the global outflow rate (Equation 15). Equation (14) is an 279 approximate law for the starved screw. The value of $\psi$ can be assessed by literature (Carrot 280 et al., 1993) or experimentally fitted. In the conveying area, the throughput is the result of a 281 transport phenomenon in the intermeshing zone and then, when this zone is fully filled, of a 282 pumping flow in the C-channel area. In the intermeshing zone, material moves forward in the axial direction a distance equivalent to the pitch for every screw revolution, whatever the operating conditions are. In the channels, material conveying is mainly due to the friction of solid polymer with both barrel and screw. Equation (15) expresses the mean velocity of matter in the fully filled zone. According to Equation 16, the axial dispersion coefficient $D_{a x}$ depends on the flow regime. The term $\alpha N-Q$ takes into account the pressure gradient influence on dispersion and the $\lambda$ correction parameter is thought to modulate the value of the axial dispersion function $D_{a x}$ by taking into account the screw channel filling.

$$
D_{a x} \frac{\partial C}{\partial z}=v \cdot\left(C-C_{i n}\right) \text { for } z=l_{t o t}
$$

$$
\frac{\partial C}{\partial z}=0 \text { for } z=0
$$

292 Tracer output concentration was estimated by numerical solving of Equation 13, boundary 293 conditions being defined by Equation 17 and 18 (finite difference approximation has been 294 used for partial derivatives). 


\section{SIMULATIONS}

298 In order to illustrate the possibilities offered by the proposed model several simulations are 299 presented in this part. It enables to simulate RTD in function of process parameters (flow rate 300 and screw speed) and geometrical parameters (screw profile and die design). Simulations 301 were performed with Matlab software (Simulink toolbox). A 100 units pulse of tracer from 302 $t=0$ to $t=2 \mathrm{~s}$ at the feeding section of the extruder was considered. All simulations presented in this paper were performed with $m=100$ spatial discretization elements.

304 Figure 4 illustrates the RTD evolution in function of process and geometrical parameters 305 within the frame of the explored experimental domain. Increasing screw speed leads to a 306 decrease of RTD pure delay and dispersion. An increasing flow rate leads also to a 307 distribution width decrease and to a more Gaussian distribution shape (Figure 4a\&b). By 308 increasing the screw pitch mean residence time increases and distribution become larger. For 309 a low screw speed, pure time-delay (delay before tracer concentration increase) increases with 310 screw pitch, whereas for a high screw speed, pure time-delay is not influenced by screw pitch.

311 Increasing restriction at the die leads to a larger distribution, but has no influence on pure 312 time-delay (Figure 4c\&d). Observed tendencies are in agreement with what is commonly 313 described in literature. These above calculations show that the influence of process and 314 geometrical parameters can be simulated thanks to the proposed model, which could be useful 315 for die and screw profile design. 
In order to work in absolutely homogeneous conditions, experiments with Laminaria digitata

320 were, as explained before, carried out during a second run through the extruder. Hence,

321 alkaline reaction had already occurred and only mechanical properties where involved in

322 material flow.

323 Model validation was performed in the case of a constant viscosity along the screws with

324 experimental data from two different extrusion applications, reactive extrusion of seaweeds

325 for alginate extraction and LDPE (low density polyethylene) extrusion. Seaweed extrusion

326 experiments are described below and polymer extrusion data were obtained from Puaux et al.

327 (2000). Assumption of a constant viscosity is maintained for polymer experiments even if

328 authors are perfectly aware that this assumption appears extremely limitative.

$330 \quad$ 4.1. Seaweeds extrusion

331 4.1.1. Experimental

332 Validation for the proposed RTD model was performed with experimental data obtained from

333 a seaweed reactive extrusion application developed by Vauchel et al. (2008). A carbonatation

334 step by means of reactive extrusion is applied to extract alginate from brown seaweeds. The

335 alginate extraction protocol was adapted from the industrial process described by Pérez et al.

336 (1992).

337 All experiments were conducted on two-year-old Laminaria digitata fronds harvested in

338 Portsall, Brittany, France. The entire fronds were cut into small pieces $\left(5 \mathrm{~mm}^{2}-5 \mathrm{~cm}^{2}\right)$ by

339 means of a separator (RM70S type provided by LIMA S.A.S., Quimper, France) and stored in

340 a $2 \%(\mathrm{w} / \mathrm{w})$ formalin solution to ensure their preservation during stocking (about 4 months).

341 Before each extraction experiment, algae pieces were rinsed with distilled water in order to

342 eliminate any formalin present, immersed in a $0.5 \mathrm{M} \mathrm{H}_{2} \mathrm{SO}_{4}$ solution for at least one night

343 (stored at $4{ }^{\circ} \mathrm{C}$ ), and rinsed again with distilled water to eliminate excess acid. The alkaline 
extraction step was conducted in a corotative twin-screw extruder (BC21 type provided by

345 Clextral, Firminy, France) equipped with a $4 \mathrm{~mm}$ diameter and $5 \mathrm{~cm}$ long cylindrical die.

346 Algae pieces were introduced in the hopper and the feed rate was regulated by means of a 347 feed pump. An external volumetric pump was used to supply the extruder with a 5\% (w/w)

$348 \mathrm{Na}_{2} \mathrm{CO}_{3}$ solution. As alginate starts to degrade at $40^{\circ} \mathrm{C}$, the barrel temperature was maintained 349 at about $20^{\circ} \mathrm{C}$ thanks to a circulating cooling water system.

350 Two different screw profiles were used, a simple one composed of decreasing direct pitch 351 screw elements and a small reverse screw element (profile 1 in Figure 3a) and a restrictive 352 one including two kneading discs sections (profile 2 in Figure 3d). As Algae feed rate to 353 reactive solution feed rate ratio $(r)$ influences process efficiency, two different values for this 354 parameter were also considered, $r=1$ and $r=3$. They correspond to the boundary values of $r$ for 355 the experimental area where seaweeds extrusion operates. All experiments were undertaken 356 for a fixed screw speed and a global feed rate of respectively $300 \mathrm{rpm}$ and $4 \mathrm{~kg} \cdot \mathrm{h}^{-1}$.

357 Experimental RTD were obtained by injecting a tracer at the feeding section of the extruder 358 and quantifying tracer concentration at the die exit. A red food colouring agent (E124) was 359 used. One $\mathrm{mL}$ of a $2 \mathrm{~g} . \mathrm{L}^{-1}$ solution of this colouring agent was injected at $t=0$ at the feeding 360 section with a syringe. Extrudate was collected in several samples, each one corresponding to 361 a 10s time interval. Each sample was diluted in water and centrifuged at $10000 \mathrm{~g}$ for 10 362 minutes (centrifuge KR22i Jouan S.A.S, Saint-Herblain, France). Supernatant tracer content 363 was quantified by measuring absorbance at 507nm (UV-vis spectrophotometer UV2 Unicam, 364 Cambridge, UK). It appeared that almost all tracer injected at the feeding section is recovered 365 in the outgoing material supernatant during the experiments.

\subsubsection{Simulation results}

367 Few assumptions were adopted concerning some elements of the restrictive screw profile to 368 perform simulations. Grooved reverse pitch elements and kneading elements were replaced by 
reverse screw elements $(25 \mathrm{~mm}$ long with a $16.6 \mathrm{~mm}$ pitch and $50 \mathrm{~mm}$ long with a $25 \mathrm{~mm}$ pitch respectively). For each feed rates ratio, one of the two experimental RTD was used to adjust

371 values of the parameter $\lambda_{1}$. Parameter $\lambda_{2}$ was defined in function of $\lambda_{1}: \frac{\lambda_{2}}{\lambda_{1}}=10$, as axial 372 dispersion is lower in the conveying zones than in the fully filled zones. This ratio value was 373 adopted because it appeared as a good compromise between a too low value that would 374 deteriorate the adjustment quality (minimization by the least squares method) and a too high 375 value that would raise numerical problems during the resolution. Hence, for $r=1, \lambda_{l}=100 \mathrm{~m}^{-1}$ 376 and $\lambda_{2}=1000 \mathrm{~m}^{-1}$ were used and for $r=3, \lambda_{1}=160 \mathrm{~m}^{-1}$ and $\lambda_{2}=1600 \mathrm{~m}^{-1}$. In Figure 7, curves $a$ 377 and $b$ correspond to parameters adjustment and curves $c$ and $d$ to simulations performed by 378 means of the proposed model.

379 Simulation results presented in Figure 5 globally show that the proposed model provides good 380 predictions of experimental RTD curves. Experimental data clearly highlight the influence of 381 feed rates ratio on flow in the extruder. Increasing $r$ (the flow rate being constant) leads to an 382 increase of the mean residence time and a wider distribution for both screw profiles. Then, 383 changing screw profile also induces flow modifications in the extruder. Screw profile 2 being more restrictive, the mean residence time increases and distribution is wider than for screw profile 1 . The observed evolution was satisfactorily simulated by the proposed model.

\subsection{Polymer extrusion}

388 Data from literature have been used so as to validate the proposed model for another type of 389 material. The work published by Puaux et al. (2000) has been chosen because all information 390 needed to perform simulations were mentioned (screw profile, die design, material 391 properties). RTD evolution was assessed for different screw speed values in the case of low 392 density polyethylene extrusion with a BC21 type extruder (Clextral S.A.S., Fiminy, France). 393 The material being different from the previous case, value of $\lambda_{l}$ parameter was modified. It 
was adjusted according to one of the four known experimental RTD curves: for $N=150 \mathrm{rpm}$, $\lambda_{l}=150 \mathrm{~m}^{-1}$. Screw profile used by Puaux et al. (2000) is described in Figure 6 (profile 3). Figure 7 presents experimental data and predictions from the proposed model. Predictions were globally close to experimental RTD, with a correctly simulated shape. However, some imprecisions can be noticed. Distribution width was a little underestimated and predictions were a little time-lagged, particularly for high screw speed values. Despite these imprecisions, this second validation case confirms the ability of the proposed model to simulate and predict RTD curves from process and geometrical parameters even with the assumption of a constant viscosity along the screw.

\section{CONCLUSION}

In this paper, a new model is proposed to predict residence time distribution in fully

intermeshing co-rotating twin-screw extruders, taking into account control parameters (screw speed and flow rate) and geometrical parameters (screw profile and die design). Possibilities offered by the proposed model were illustrated by simulations for various operating and

410 geometrical conditions. Validation was performed for two different applications, seaweeds extrusion and polymer material extrusion. It showed the model ability to predict RTD for various kinds of extruded materials. The originality of the proposed model lies in its ability to predict RTD after adjusting only one parameter $\left(\lambda_{1}\right)$ thanks to a unique experimental RTD

414 curve. Once parameters adjustment is performed, RTD can be predicted for different 415 operating conditions (screw speed and feed rate) and different geometrical configurations 416 (screw profile and die design).

417 The proposed RTD model could be improved by adding several extensions. It could be 418 extended to the reactive case by coupling reaction kinetics to the equation describing spatio 
temporal tracer concentration evolution (Equation 13). It would also be possible, provided

420 other assumptions are made, to adapt the structure of equation 10 to take into account the case

421 of an evolving viscosity along the screw channel. And if reaction advancement and viscosity

422 are linked, it would also be possible to take it into account if the relation is correctly

423 formalized. The coupling between kinetic model of alginate extraction and flow model will be

424 discussed in a forthcoming paper. The extended model could be a useful tool to help

425 optimizing reactive extrusion applications.

\section{REFERENCES}

429 Ainser, A., 1996. Etudes des écoulements et réactions chimiques en extrudeuse bi-vis.

430 Approche expérimentale : utilisation de la distribution des temps de séjour. $\mathrm{PhD}$ thesis,

431 Université de Saint-Etienne.

432 Baron, R., 1995. Modélisation et commande d'un procédé d'extrusion de pulpe de poisson.

433 PhD thesis, Université Paris Sud.

434 Berzin, F., Hu, G.H., 2004. Procédés d'extrusion réactive. Techniques de l'Ingénieur, 435 AM3654.

436 Carrot, C., Guillet, J., May, J.F., Puaux, J.P., 1993. Modeling of the conveying of solid 437 polymer in the feeding zone of intermeshing co-rotating twin screw extruders. Polymer 438 Engineering and Science, 33 (11), 700-708.

439 Dufaure, C., Mouloungui, Z., Rigal, L., 1999. A twin-screw extruder for oil extraction: II. 440 Alcohol extraction of oleic sunflower seeds. Journal of the American Oil Chemists' Society, $441 \quad 76,1081-1086$. 
442 Ganzeveld, K.J., Janssen, L.P.B.M., 1993. Twin screw extruders as polymerization reactors 443 for free radical homo polymerization. The Canadian Journal of Chemical Engineering, 71, $444 \quad 411-418$

445 Janssen, L.P.B.M., Hollander, R.W., Spoor, M.W., Smith, J.M., 1979. Residence time 446 distributions in a plasticating twin screw extruder. AIChE Journal, 25, 345-351.

447 N'Diaye, S., Rigal, L., Larocque, P., Vidal, P.F., 1996. Extraction of hemicelluloses from 448 poplar, Populus tremuloides, using an extruder-type twin-screw reactor: a feasibility study. 449 Bioresource Technology, 57, 61-67.

450 Pérez, R., Kaas, R., Campello, F., Arbault, S., Barbaroux, O., 1992. La culture des algues 451 dans le monde. Ifremer, Plouzané, France.

452 Perrin, J.L., De Choudens, C., 1996. Extrusion technology in paper pulp processing. In: 453 Proceedings of the Conference on ' 40 years of twin-screw extrusion at Clextral', Firminy, 454 France, 8-10 October 1996.

455 Prat, L., Guiraud, P., Rigal, L., Gourdon, C., 1999. Two phase residence time distribution in a 456 modified twin-screw extruder. Chemical Engineering and Processing, 38, 73-83.

457 Prat, L., Guiraud, P., Rigal, L., Gourdon, C., 2002. A one dimensional model for the 458 prediction of extraction yields in a two phases modified twin-screw extruder. Chemical 459 Engineering and Processing, 41, 743-751.

460 Puaux, J.P., Bozga, G., Ainser, A., 2000. Residence time distribution in a corotating twin461 screw extruder. Chemical Engineering Science, 55, 1641-1651.

462 Puaux, J.P., Cassagnau, P., Bozga, G., Nagy, I., 2006. Modeling of polyurethane synthesis by 463 reactive extrusion. Chemical Engineering and Processing, 45, 481-487.

464 Rouilly, A., Jorda, J., Rigal, L., 2006. Thermo_mechanical processing of sugar beet pulp. I. 465 Twin-screw extrusion process. Carbohydrate Polymers, 66, 81-87. 
467 Reinhold Company, New York.

468 Vauchel, P., Baron, R., Kaas, R., Arhaliass, A., Legrand, J., 2008a. A new process for 469 extracting alginates from Laminaria digitata : reactive extrusion. Food and Bioprocess

470 Technology : an International Journal, 1, 297-300.

471 Vauchel, P., Le Roux, K., Kaas, R., Arhaliass, A., Baron, R., Legrand, J., 2008b. Kinetics

472 modeling of alginate alkaline extraction from Laminaria digitata. Bioresource Technology, $473 \quad 100,1291-1296$.

474

\section{Figure captions}

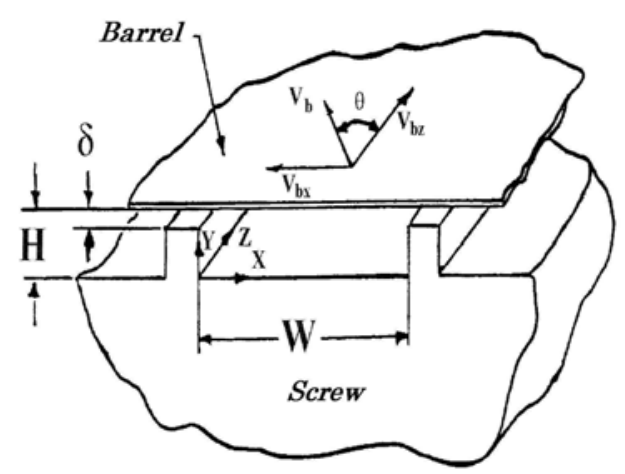

476

477 Figure 1. The unrolled channel and the moving plane barrel (Tadmor and Klein, 1970).

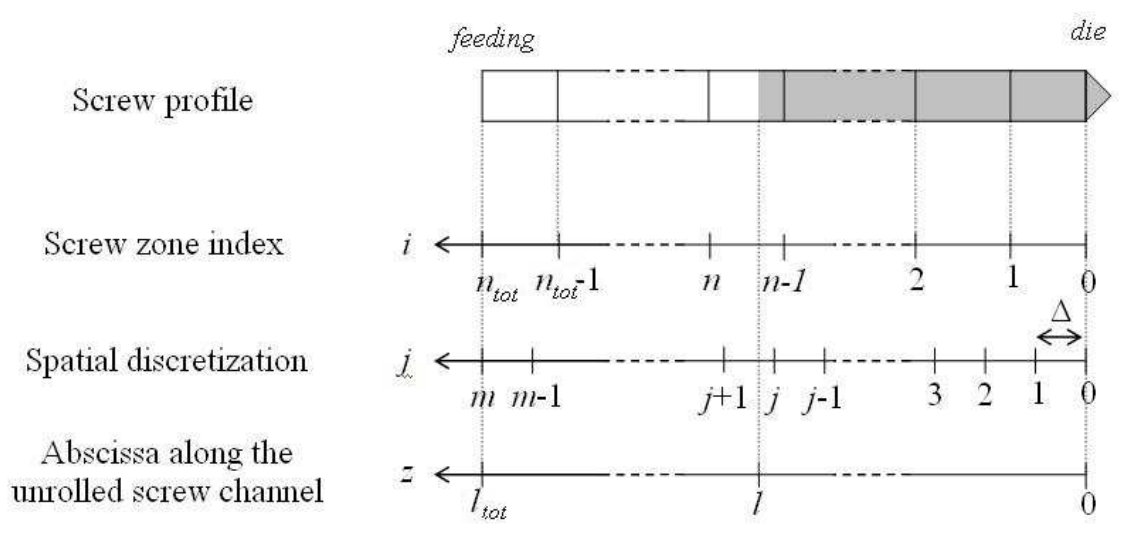

479 Figure 2. Screw profile, screw zone index, spatial discretization and abscissa along the 480 unrolled screw channel. 


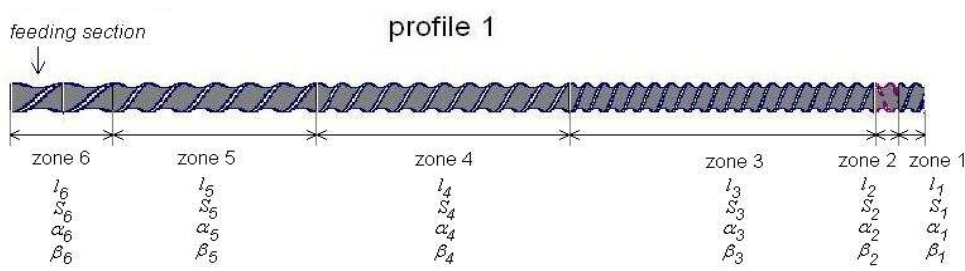

$3 \mathbf{b}$
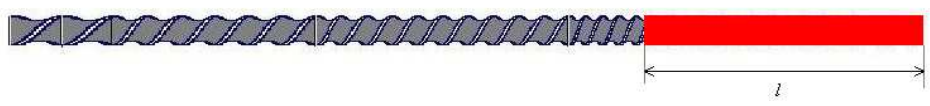

$3 c$

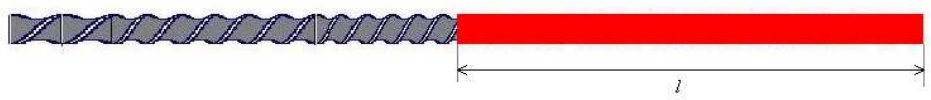

feeding section

$$
\downarrow
$$

profile 2

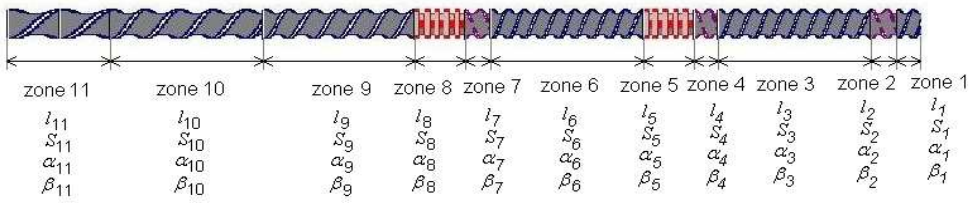

$3 e$

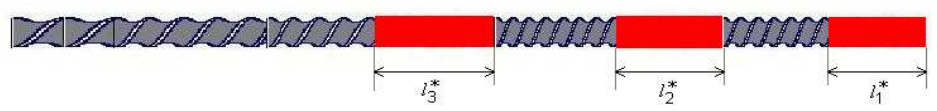

481

482 Figure 3. Screw profiles used for seaweeds extrusion experiments. (a) screw profile 1 and

483 geometrical parameters associated; (b) case where $l<\left(l_{3}+l_{2}+l_{1}\right)$ with profile 1 ; (c) case where

$484 \quad\left(l_{3}+l_{2}+l_{1}\right) \leq l<\left(l_{4}+l_{3}+l_{2}+l_{1}\right)$ with profile 1 ; (d) screw profile 2 and geometrical parameters

485 associated; (e) case of three different fully filled zones with profile 2 (and with $l_{3}^{*}<\left(l_{9}+l_{8}+l_{7}\right)$ ).
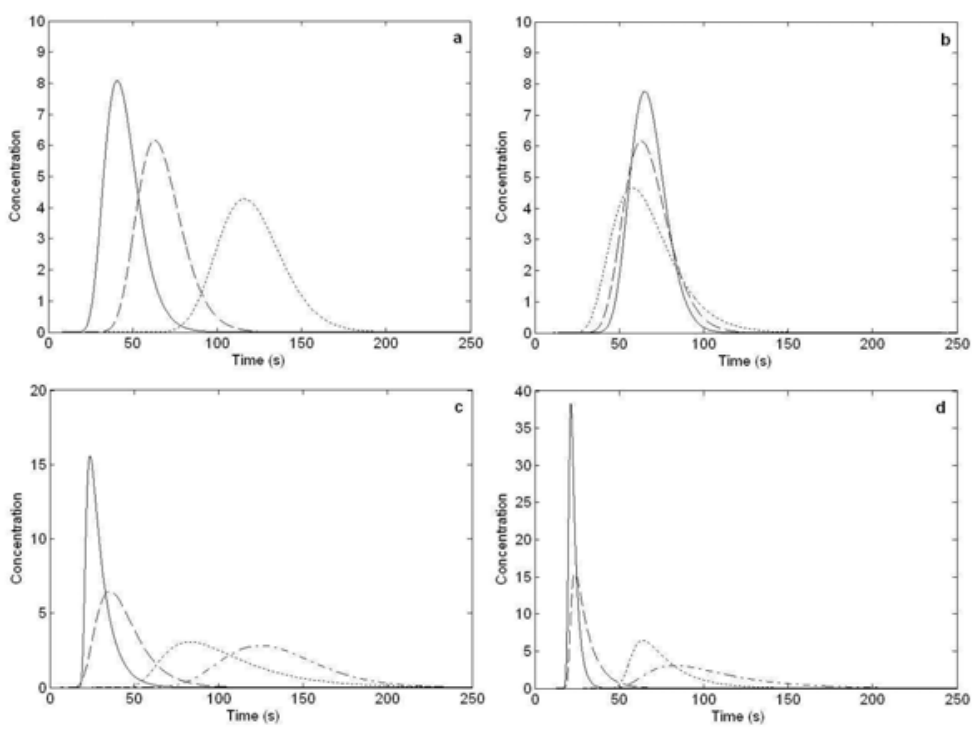
487

488

489

490

491

492

493

494 495

496

497

498

499

500

501

Figure 4. RTD simulations for different process and geometrical parameters. (a) different values of screw speed : (-) N=600rpm; (- $) N=400 \mathrm{rpm} ;(---) N=200 \mathrm{rpm}\left(Q=5 \mathrm{~kg} \cdot \mathrm{h}^{-1}\right.$; $\left.p=50 \mathrm{~mm} ; K=7 \cdot 10^{-11} \mathrm{~m}^{3}\right)$. (b) different values of flow rate: $(---) Q=3 \mathrm{~kg} \cdot \mathrm{h}^{-1} ;(--) Q=5 \mathrm{~kg} \cdot \mathrm{h}^{-1}$; (一) $Q=7 \mathrm{~kg} \cdot \mathrm{h}^{-1}\left(N=400 \mathrm{rpm} ; p=50 \mathrm{~mm} ; K=7 \cdot 10^{-11} \mathrm{~m}^{3}\right)$. (c) different values of screw pitch and screw speed : (---) N=200rpm and $p=25 \mathrm{~mm} ;(---) N=200 \mathrm{rpm}$ and $p=50 \mathrm{~mm} ;(-)$ $N=600 \mathrm{rpm}$ and $p=25 \mathrm{~mm} ;(--) N=600 \mathrm{rpm}$ and $p=50 \mathrm{~mm}\left(Q=5 \mathrm{~kg} \cdot \mathrm{h}^{-1} ; K=7 \cdot 10^{-11} \mathrm{~m}^{3}\right) .(\mathrm{d})$ different values of die restriction coefficient and screw speed : (-) $N=600 \mathrm{rpm}$ and $K=1,5.10^{-}$ ${ }^{10} \mathrm{~m}^{3} ;(--) N=600 \mathrm{rpm}$ and $K=7.10^{-11} \mathrm{~m}^{3} ;(---) N=200 \mathrm{rpm}$ and $K=1,5.10^{-10} \mathrm{~m}^{3} ;(---)$ $N=200 \mathrm{rpm}$ and $K=7 \cdot 10^{-11} \mathrm{~m}^{3}\left(Q=5 \mathrm{~kg} \cdot \mathrm{h}^{-1} ; p=25 \mathrm{~mm}\right)$.
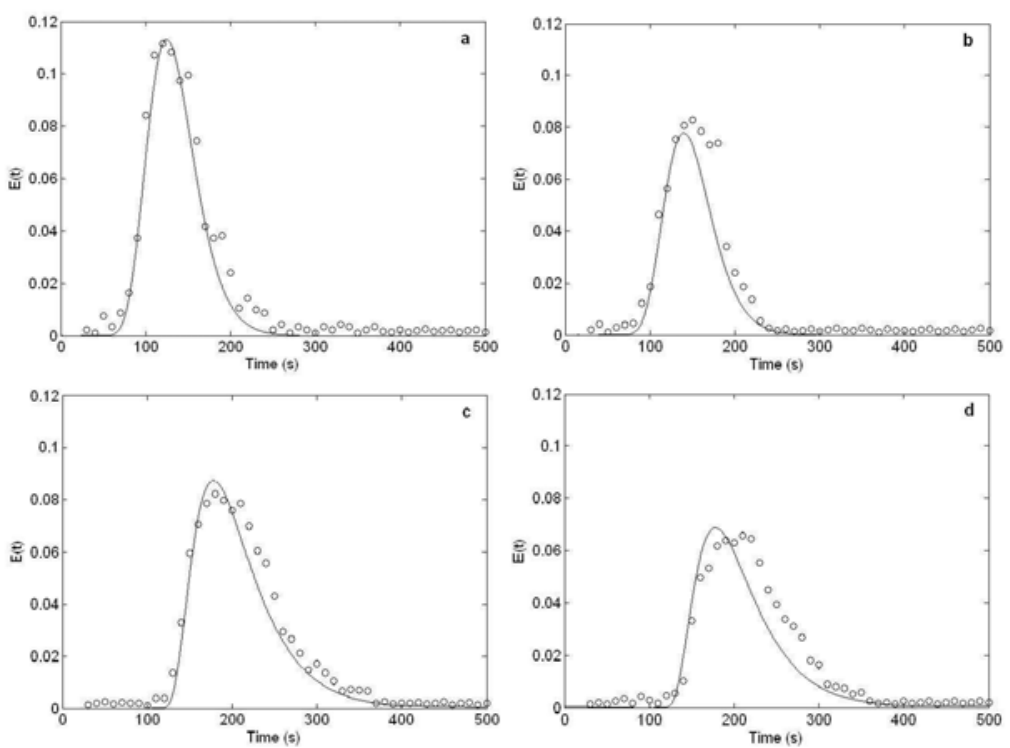

Figure 5. Model validation for seaweeds extrusion for two different screw profiles and feed rates ratios. $(\mathrm{O})$ experimental data; $(-)$ model prediction. Parameters adjustment : (a) screw profile 1 and $r=1$; (b) screw profile 1 and $r=3$. Simulations: (c) screw profile 2 and $r=1$; (d) screw profile 2 and $r=3$.

feeding section

$\downarrow$

profile 3

502 Figure 6. Screw profile 3 used by Puaux et al. (2000) for LDPE extrusion. 


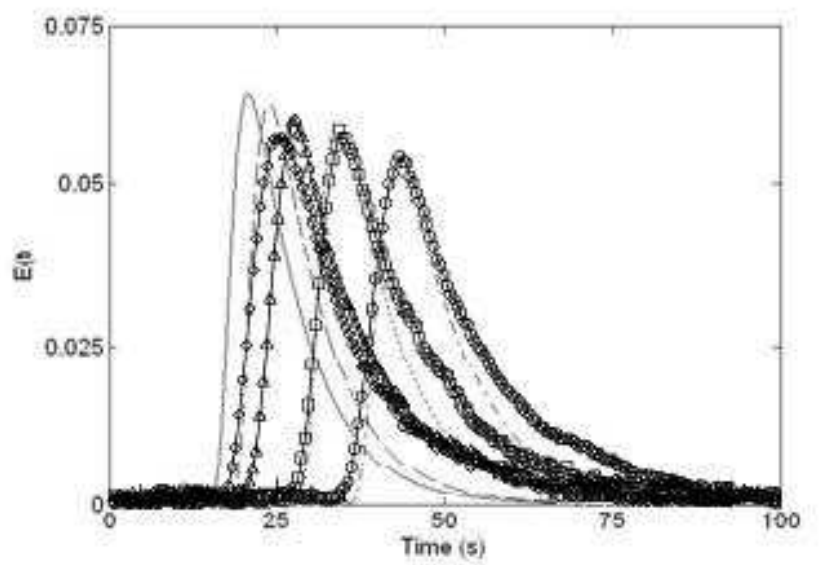

504 Figure 7. Model validation for polymer extrusion. Experimental data: $(\diamond) N=400 \mathrm{rpm} ;(\Delta)$

$505 N=300 \mathrm{rpm} ;$ ( ) N=200rpm; (O) N=150rpm. Model prediction: (-) N=400rpm; (- -)

$506 \quad N=300 \mathrm{rpm} ;(---) N=200 \mathrm{rpm} ;(---) N=150 \mathrm{rpm}$. 